\title{
Isolation and genotyping of Candida albicans involved in vaginal candidiasis among pregnant women in Sulaymaniyah and Erbil cities
}

\begin{abstract}
Background and objective: Vaginal candidiasis is a common infection among pregnant women. The current study aimed to investigate the etiologic species of vaginal candidiasis and the genotypes of $C$. albicans isolated from vaginal samples among pregnant women in the Kurdistan region of Iraq.
\end{abstract}

Methods: Vaginal swabs were collected from pregnant women admitted to the Maternity hospital in Sulaymaniyah and Erbil cities from March 2016 to December 2018. Candida isolates were identified on CHROMagar medium, then confirmed with PCR depending on ITS region. All C. albicans isolates were confirmed using chitin synthase gene (CHS1) and subjected to genotypic analysis based on the transposable intron in 25S rDNA with using CA25S and CA-INT primers. DNA sequencing of 25S rDNA region was done by using CA and CA-INT primers.

Results: Among 340 women tested, 114 (33.53\%) were positive for vaginal candidiasis. Five Candida species were identified, where they are C. albicans (56.14\%), C. glabrata (24.56\%), C. kefyr (11.40\%), C. tropicalis (5.27\%) and C. krusei (2.63\%). It has been found that $C$. albicans significantly $(P<0.01)$ higher than non-albicans species. The genotypes $A$ (450 bp), B (840 bp), and C (450 and $840 \mathrm{bp)} \mathrm{of} \mathrm{C.} \mathrm{albicans} \mathrm{were} \mathrm{detected.} \mathrm{The} \mathrm{Genotype}$ A (54.69\%) was the most prevalent, followed by Genotype B (34.38\%) and Genotype C (10.94\%). In regards to genetic variation, genotypes A and B were more similar compared to genotype C.

Conclusion: The current study revealed a high prevalence of vaginal candidiasis with different genotypes of $C$. albicans among pregnant women. Therefore, it is worth considering a vaginal swab culture with clinical symptoms during the diagnosis of vaginal candidiasis.

Keywords: Candida; Genotypes; Vulvovaginitis; Candidiasis; Prevalence.

\section{Introduction}

Candida species are the most common cause of fungal infections, leading to a range of life-threatening invasive diseases such as bloodstream candidiasis to non-life-threatening mucocutaneous candidiasis such as genitourinary candidiasis, vulvovaginal candidiasis, and oropharyngeal candidiasis. ${ }^{1}$ Genital involvement in women is one of the most common presentations due to Candida. ${ }^{2}$ Vulvovaginal candidiasis (VVC) is caused by the overgrowth of Candida species in the vagina and is characterized by curd-like vaginal discharge, itching, and erythema ${ }^{3}$ and has increased dramatically in recent years as a worldwide health problem and affects millions of women annually. ${ }^{2}$ It is estimated that $70 \%-75 \%$ of women of childbearing age worldwide experience at least one episode during their lifetime, and $5 \%-10 \%$ of women with a first episode of VVC experience frustrating recurrent infections. VVC is rare before puberty. Its incidence increases dramatically in the second decade of life, coinciding with the onset of sexual activity. ${ }^{4}$ Some of the factors that predispose women to vaginal

${ }^{1}$ Department of Biology, College of Science, University of Sulaimani, Sulaymaniyah, I raq.

* Correspondence: khattab.mustafa@univsul.edu.iq 
candidiasis are changing in $\mathrm{pH}$, use of oral contraceptives, tight clothing, and personal hygiene. ${ }^{5}$ However, it is reported that vaginal candidiasis is common during pregnancy due to the altered $\mathrm{pH}$ and sugar content of vaginal secretions. Also, increasing the estrogen level during pregnancy produces more glycogen in the vagina, which directly affects yeast cells, causing them to grow faster and stick more easily with the walls of the vagina. ${ }^{6}$ It has been estimated that nearly up to $40 \%$ of pregnant women worldwide may have vaginal colonization by Candida species. ${ }^{5-7}$ Candida albicans is the most common causative pathogen of candidiasis. Recently, other Candida species known as non-albicans, such as C. glabrata, C. tropicalis, C. parapsilosis, C. krusei and C. dubliniensis have emerged as important opportunistically infectious fungi. ${ }^{8-10}$ Thus, it is well known that $C$. albicans is the leading agent of vaginal fungal infections and other gynaecological disorders. Almost $80-90 \%$ of VVC is caused by $C$. albicans except that only a minority of cases (10-20\%) is caused by non-C. albicans species. ${ }^{11}$ Genetic variability and key virulence factors such as adhesion capacity, biofilm development, cell surface hydrophobicity, morphological transition, and production of hydrolytic enzymes by $C$. albicans are recognized essential steps in the process of Candida colonization and subsequent infection. ${ }^{12}$ Heterogeneity of these virulence properties in clinical C. albicans isolates is commonly observed; hence, molecular subtyping provides a useful tool to identify pathogenic subpopulations within this species. According to the presence and the size of transposable intron region in the large ribosomal subunit 25S rDNA, C. albicans can be divided into four subtypes based on different lengths of PCR products amplified namely, genotype A (450 bp), genotype B (840 bp), genotype C (450- and 840-bp), and genotype E (1400 bp). ${ }^{13}$ Reports are indicating an association of certain C. albicans genotypes with fungal invasiveness and antifungal susceptibility. ${ }^{13}$
Thus, the current study aimed to determine the etiologic species of vaginal candidiasis and genotyping of $C$. albicans isolated from vaginal samples among pregnant women in the Kurdistan region of Iraq.

\section{Methods}

\section{Sample Collection}

Using sterile cotton tipped swabs, a total of 340 vaginal swabs were collected from symptomatic and asymptomatic pregnant women who were admitted to Maternity hospital in Sulaymaniyah and Erbil cities, Kurdistan Region, Iraq, from March 2016 to December 2018. The collection of vaginal swabs was done by a gynecologist. The swab stick was immediately replaced with its casing, labeled, and taken to the laboratory for culturing. ${ }^{14}$ The participants filled out a consent form to participate in the research, which was approved by the Research Protocol Ethics Committee of the Kurdistan Board of Medical Specialties. Concomitant to each sample, a questionnaire was completed for each participant inquiring about their age, marital state, occupation, address, symptomatic state, pregnancy trimester, history of antibiotic use, taking birth control pills, associated medical conditions during pregnancy, and presence of stress factors.

\section{Isolation and Phenotypic Identification} of Candida spp.

Candida spp. were isolated by direct plating of samples on Sabouraud dextrose agar (SDA) [Difco Laboratories Detroit, USA) supplemented with chloramphenicol (0.1 $\mathrm{mg} / \mathrm{mL}$, ASIA-Syria) and incubation at $37^{\circ} \mathrm{C}$ for 48 hours. As preliminary identification, Candida spp. were identified according tothe morphology of the colonies, which were white to cream, round, curved, soft, and smooth to wrinkled, with a characteristic yeast odor, it was overgrowing. For species identification, all Candida isolates were cultured on CHROMagar Candida (Liofilchem, Italy), a loopful of an isolate was streaked on a CHROMagar plate and incubated at $37^{\circ} \mathrm{C}$. The growth was 
checked every 24 hours until 72 hours of incubation, and the species were identified by type and color of the colonies on CHROMagar media as per 'manufacturer's instructions. ${ }^{15-17}$

\section{Molecular Identification of Candida spp.}

The molecular identification was performed for all the isolates using the primers shown in Table 1, also with a colony PCR to save material and time. Through an overnight culture, a single colony of Candida was picked then re-suspended in $40 \mu \mathrm{l} d \mathrm{dd}_{2} \mathrm{O}$. An incubation at $95^{\circ} \mathrm{C}$ for $20 \mathrm{~min}$ done to release the DNA according to Lau et al. ${ }^{18}$ and Alshahni et al. ${ }^{19}$ The DNA was purified by centrifugation at $12000 \mathrm{rpm}$ for $2 \mathrm{~min}$ then $3 \mu \mathrm{l}$ of the supernatant used as a PCR template. ITS primers were used to identify different strains of the Candida spp., ${ }^{20}$ which amplified the intergenic spacer regions (ITS) (Figure 1) of ribosomal DNA (rDNA). ${ }^{21}$ The PCR was performed using Prime Taq DNA Polymerase kit [Prime Taq Premix (2X)/ GeNet Bio) according tomanual instructions. The program of the PCR was as follows: 1 cycle of initial denaturation at $95^{\circ} \mathrm{C}$ for $5 \mathrm{~min}$, followed by 35 amplification cycles of denaturation at $95^{\circ} \mathrm{C}$ for $30 \mathrm{sec}$, annealing at $57^{\circ} \mathrm{C}$ for $30 \mathrm{sec}$ and extension at $72^{\circ} \mathrm{C}$ for $40 \mathrm{sec}$, then finalized with the final extension at $72^{\circ} \mathrm{C}$ for $5 \mathrm{~min}$. The amplicon was separated by $1 \%$ gel electrophoresis then visualized by ethidium bromide.

\section{Confirmation of the Candida albicans} identification by PCR

For more confirmation, all the amplicon with the 532bp in size (previously amplified by ITS primers) were re-analyzed by another set of primers CHS1, according toJordan. ${ }^{22}$ The conventional PCR enables to amplify a portion of a chitin synthase gene (CHS1) from C.albicans with the size of 122bp.The colony PCR was also followed as previously mentioned with the same DNA polymerase kit. The PCR program was as follows: initial denaturation for $5 \mathrm{~min}$ at $95^{\circ} \mathrm{C}$, then in 40 cycles of denaturation at $95^{\circ} \mathrm{C}$ for $30 \mathrm{sec}$, annealing at $52^{\circ} \mathrm{C}$ for $30 \mathrm{sec}$, and extension at $72^{\circ} \mathrm{C}$ for $30 \mathrm{sec}$ were adapted. The PCR was terminated with one cycle of finale extension at $72^{\circ} \mathrm{C}$ for $7 \mathrm{~min}$. The products were also visualized with the $2 \%$ gel electrophoresis and ethidium bromides.

Table 1: List of the primers used in this study.

\begin{tabular}{|c|c|c|c|}
\hline Name & Sequence $\left(5^{\prime} \rightarrow 3\right)$ & $\mathrm{Tm}^{0} \mathrm{C}$ & References \\
\hline ITS1-F & TCCGTAGGTGAACCTGCG & $57^{\circ} \mathrm{C}$ & Fujita et al. ${ }^{20}$ \\
\hline ITS4-R & TCCTCCGCTTATTGATATGC & $57^{\circ} \mathrm{C}$ & Fujita et al. ${ }^{20}$ \\
\hline CHS1-F & CGCCTCTGATGGTGATGAT & $52^{\circ} \mathrm{C}$ & Jordan $^{22}$ \\
\hline CHS1-R & TCCGGTATCACCTGGCTC & $52^{\circ} \mathrm{C}$ & Jordan 22 \\
\hline CA25S-F & CGATTCAGGGGAGGTAGTGAC & $64^{\circ} \mathrm{C}$ & Tantivitayakul et al. ${ }^{13}$ \\
\hline CA25S-R & GGTTCGCCATAAATGGCTACCAG & $64^{\circ} \mathrm{C}$ & Tantivitayakul et al. ${ }^{13}$ \\
\hline CA-INT-F & ATAAGGGAAGTCGGCAAAATAGATCCGTAA & $70^{\circ} \mathrm{C}$ & Tantivitayakul et al. ${ }^{13}$ \\
\hline CA-INT-R & CCTTGGCTGTGGGTTTCGCTAGATAGTAGAT & $70^{\circ} \mathrm{C}$ & Tantivitayakul et al. ${ }^{13}$ \\
\hline
\end{tabular}

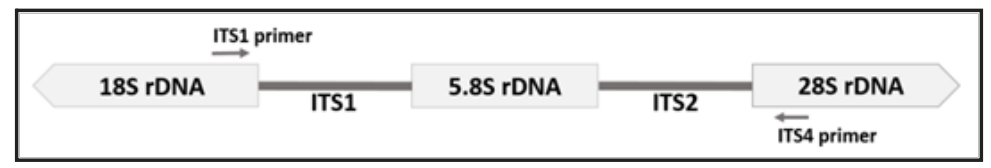

Figure 1: Positions of the amplified region by both ITS1 and ITS4 primers on the intergenic spacer regions (ITS) of ribosomal DNA (rDNA). 
https:/ / doi.org/ 10.15218/ zjms.2021.012

Nested PCR for Genotypes Detection

Different genotypes of C.albicans were detected based on the transposable intron in 25S rDNA; for this purpose, the nested PCR method was followed using CA25S primers in the first round of the PCR. The method was started with one cycle of the initial denaturation at $95^{\circ} \mathrm{C}$ for $5 \mathrm{~min}$. The PCR was continued with the 35 cycles of denaturation at $95^{\circ} \mathrm{C}$ for $30 \mathrm{sec}$ and annealing at $64^{\circ} \mathrm{C}$ for $30 \mathrm{sec}$ and extension at $72^{\circ} \mathrm{C}$ for $40 \mathrm{sec}$, then the final extension at $72^{\circ} \mathrm{C}$ for $5 \mathrm{~min}$ were finalized the PCR. The amplicon of the first round was used as a template of the second PCR with the same program with the exception of using CA-INT primers in the annealing temperature at $70^{\circ} \mathrm{C}$.

\section{Sequencing}

The sequencing was done (Macrogen Company, Seoul, South Korea) for some of the samples amplified by CA25S and CA-INT forward and reversed primers (10pmol).

\section{Statistical Analysis}

Chi-square test was performed using the statistical package for the social sciences software (version 22.0), and differences were considered significant at $P<0.01$.

\section{Results}

\section{Prevalence of Candida Species}

A total of 340 vaginal swabs were collected from pregnant women and reported in the present study. Of these, 114 (33.53\%) women were positive for vaginal candidiasis, and 226 (66.47\%) women were negative for vaginal candidiasis. All isolates were identified on CHROMagar Candida medium, then confirmed with PCR depending on ITS region, which is presented in Figure 2. Thus, as listed in Table 2, five Candida species were identified between all isolates, where they are C. albicans, C. glabrata, C. kefyr, C. tropicalis and $C$. krusei. It has been revealed that the highest frequency of vaginal candidiasis of pregnant women was caused by C. albicans (56.14\%), which is significantly $(P<0.01)$ higher than non-albicans species, where they are C. glabrata $(24.56 \%)$, C. kefyr $(11.40 \%)$, C. tropicalis $(5.27 \%)$ and C. krusei (2.63\%).

Table 2: Distribution and characteristics of isolated Candida species.

\begin{tabular}{lcccc}
\hline $\begin{array}{l}\text { Candida } \\
\text { Species }\end{array}$ & $\begin{array}{c}\text { Number of } \\
\text { Isolates }\end{array}$ & $\begin{array}{c}\text { Percentage } \\
(\mathbf{\%})\end{array}$ & $\begin{array}{c}\text { Color on } \\
\text { CHROMagar }\end{array}$ & $\begin{array}{c}\text { Fragment size found by PCR } \\
\text { with ITS1-ITS4 primers }\end{array}$ \\
\hline C. albicans & 64 & 56.14 & Light green & $532 \mathrm{bp}$ \\
C. glabrata & 28 & 24.56 & Pinkish purple & $874 \mathrm{bp}$ \\
C. kefyr & 13 & 11.40 & Dark pink & $722 \mathrm{bp}$ \\
C. tropicalis & 6 & 5.27 & Metallic blue & $521 \mathrm{bp}$ \\
C. krusei & 3 & 2.63 & Rose pink & $500 \mathrm{bp}$ \\
Total & $\mathbf{1 1 4}$ & $\mathbf{1 0 0}$ & & \\
\hline
\end{tabular}

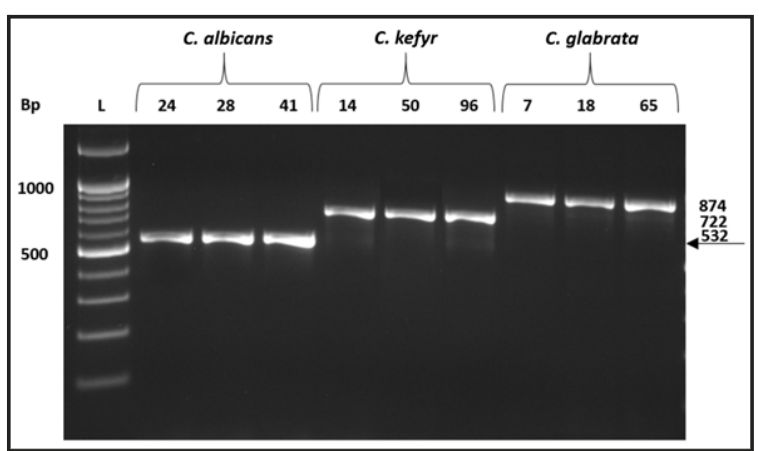

Figure 2: An example of Candida spp. Identification showing three species, which were C. albicans (532bp), C. kefyr (722bp) and C. glabrata (874bp). The marker was 100bp DNA [GeNet Bio]. 
https:/ / doi.org/ 10.15218/ zjms.2021.012

Identification and Genotyping Analysis it is shown in Figure 4. It has been found

\section{of Candida albicans}

The identification of all $C$. albicans isolates were confirmed with PCR depending on the chitin synthase gene (CHS1), as shown in Figure 3, the fragment size was 122bp. Then all C. albicans isolates were subjected to genotypic analysis based on the transposable intron in 25S rDNA with using CA25S and CA-INT primers as that $C$. albicans isolates belong to three genotypes, where are genotype A (450 bp), genotype B (840 bp), and genotype C (450 and $840 \mathrm{bp}$ ). Results showed that out of 64 isolates, the Genotype A was the most prevalent with 35 isolates (54.69\%), followed by Genotype B, with 22 isolates (34.38\%), and Genotype C, with 7 isolates (10.94\%) (Figure 5).

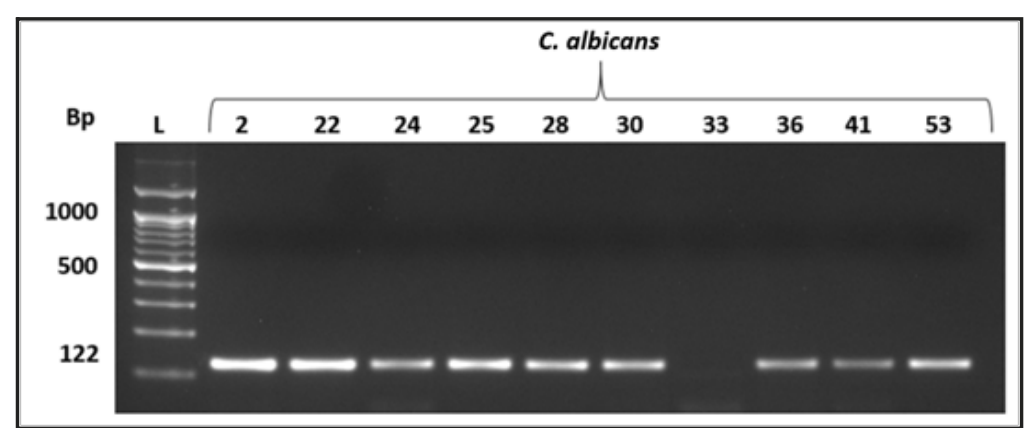

Figure 3: Confirmation of the $C$. albicans identification with different primers $\mathrm{CHS} 1$ (122bp). The marker was 100bp DNA [GeNet Bio].

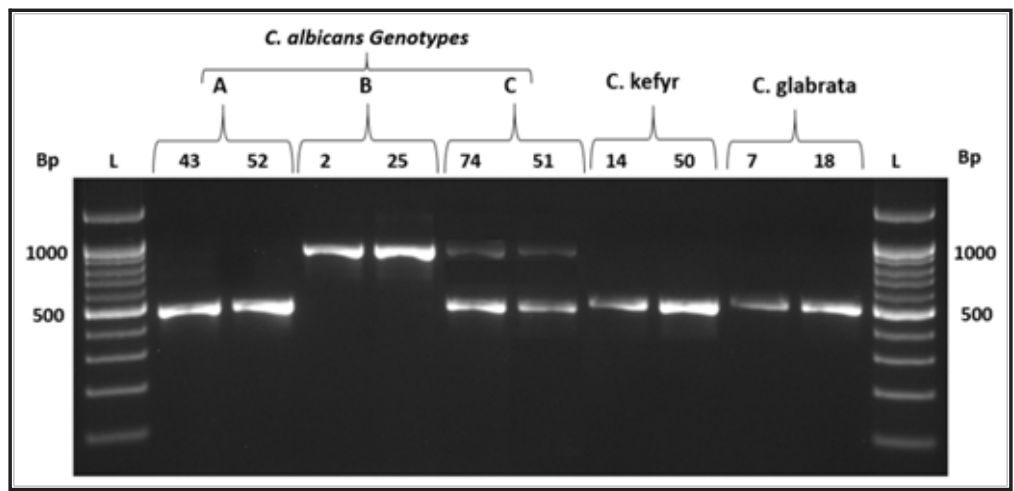

Figure 4: Detection of three genotypes of C. albicans.

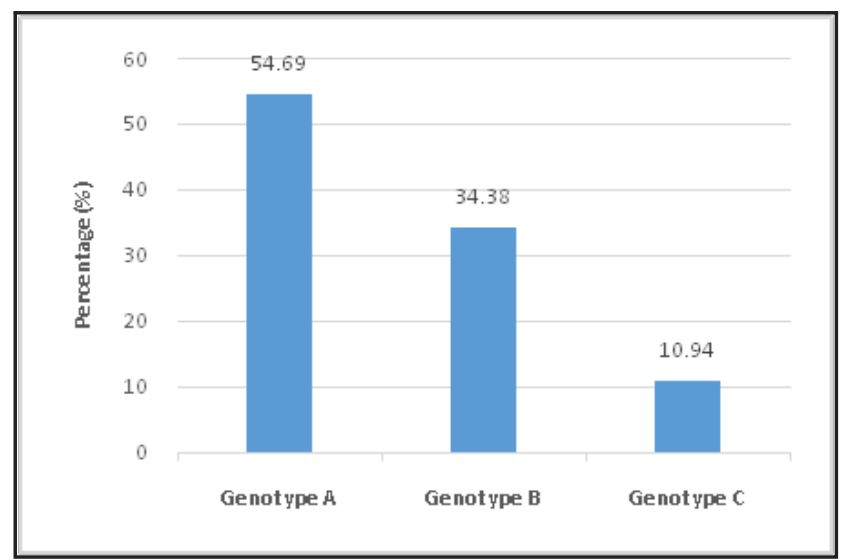

Figure 5: The percentages of C. albicans genotypes. 


\section{Phylogenetic analysis}

The evolutionary history was inferred by using the Maximum Likelihood method based on the Kimura 2-parameter model. The tree with the highest log likelihood $(-418.7355)$ is shown. The percentage of trees in which the associated taxa clustered together is shown next to the branches. Initial tree(s) for the heuristic search were obtained automatically by applying Neighbor-Join and BioNJ algorithms to a matrix of pairwise distances estimated using the Maximum Composite
Likelihood (MCL) approach and then selecting the topology with superior log likelihood value. The tree is drawn to scale, with branch lengths measured in the number of substitutions per site. The analysis involved ten nucleotide sequences. Codon positions included were 1st+2nd+3rd+Noncoding. All positions containing gaps and missing data were eliminated. There was a total of 271 positions in the final dataset. Evolutionary analyses were conducted in MEGA7.

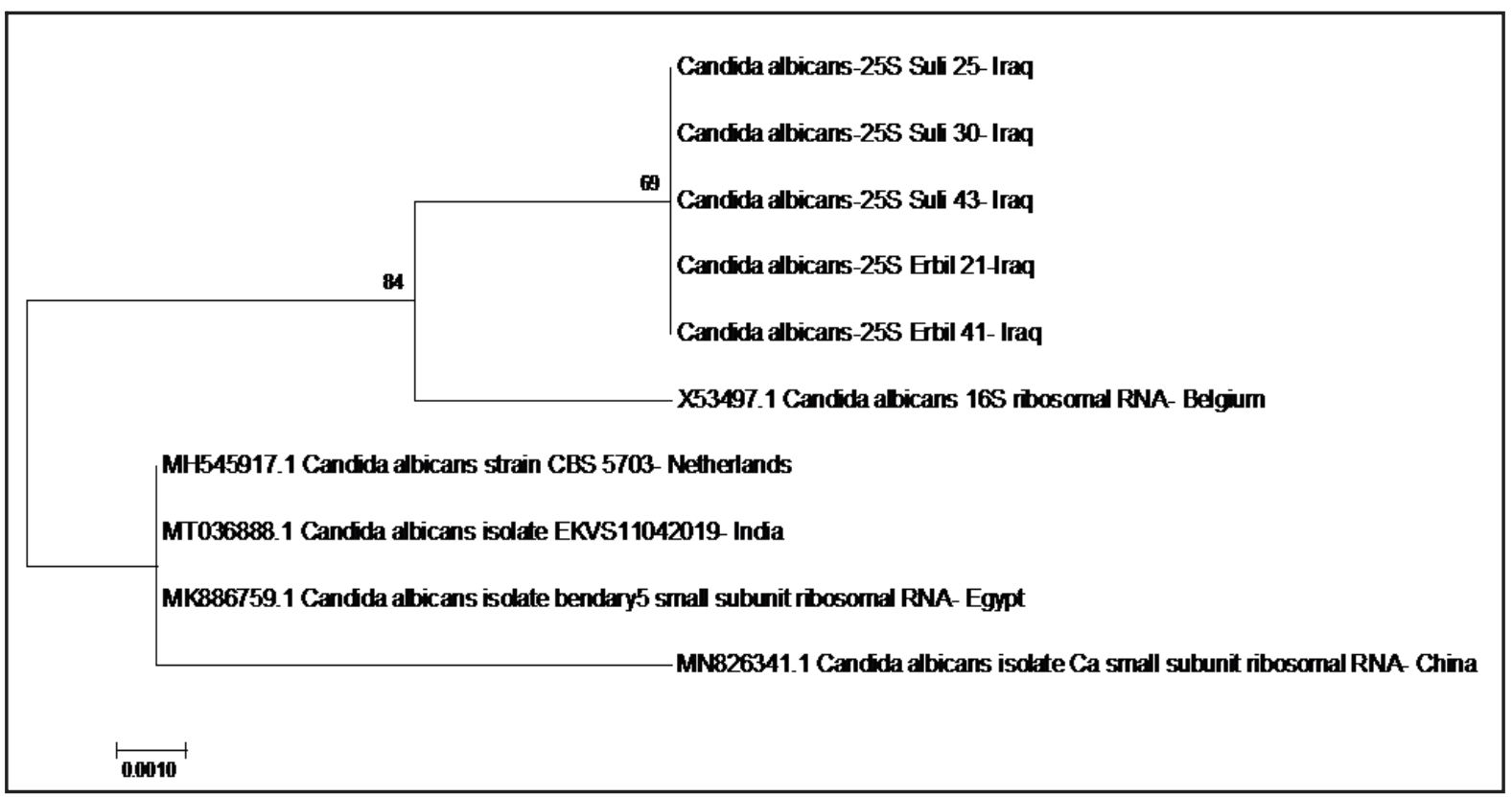

Figure 6: Molecular Phylogenetic analysis by Maximum Likelihood method of the $25 \mathrm{~S}$ region. 
The evolutionary history was inferred by using the Maximum Likelihood method based on the Kimura 2-parameter model. The bootstrap consensus tree inferred from 500 replicates is taken to represent the evolutionary history of the taxa analyzed. Branches corresponding to partitions reproduced in less than 50\% bootstrap replicates are collapsed. The percentage of replicate trees in which the associated taxa clustered together in the bootstrap test (500 replicates) are shown next to the branches. Initial tree(s) for the heuristic search were obtained automatically by applying Neighbor-Join and BioNJ algorithms to a matrix of pairwise distances estimated using the Maximum Composite Likelihood (MCL) approach and then selecting the topology with superior log likelihood value. The analysis involved six nucleotide sequences. Codon positions included were $1 \mathrm{st}+2 \mathrm{nd}+3 \mathrm{rd}+$ Noncoding. All positions containing gaps and missing data were eliminated. There wasa total of 439 positions in the final dataset. Evolutionary analyses were conducted in MEGA7.

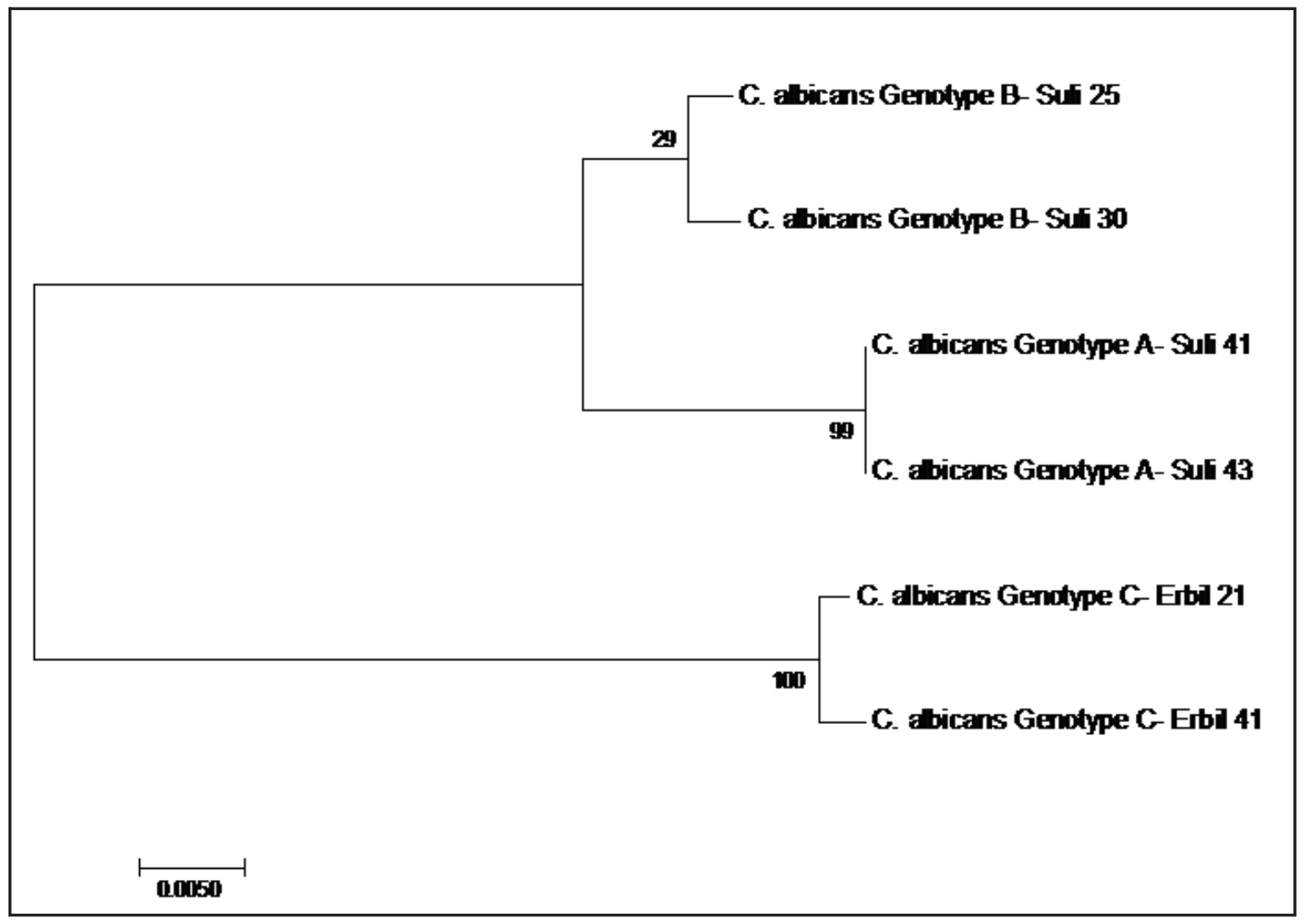

Figure 7: Molecular Phylogenetic analysis by Maximum Likelihood method of the three genotypes. 


\section{Discussion}

It is well known that Candida species is an opportunistic pathogen associated with vulvovaginal candidiasis in women, which varies from one population to another, and pregnancy is one of the risk factors. In the current study, the prevalence rate of vulvovaginal candidiasis was $33.53 \%$ between participated pregnant women. This result is lower than that reported in Lebanon (39\%), ${ }^{7}$ Pakistan (38\%), ${ }^{6}$ and India (42.37\%). ${ }^{23}$ However, another study reported a lower prevalence rate $(29 \%)$ of VVC in Iraq. ${ }^{24}$ This variation of VVC rate in pregnant women may be related to increased risk factors during pregnancy such as immunologic alterations, increased estrogen levels, and increased vaginal glycogen production mechanism. ${ }^{25}$ Five Candida species were isolated in the present study, C. albicans was the predominant species (56.14\%), followed by C. glabrata (24.56\%), C. kefyr (11.40\%), C. tropicalis (5.27\%), and C. krusei $(2.63 \%)$. These results are inconsistent with previous studies, where they stated that vaginal infection with $C$. albicans is predominated over other species. Nelson et al. $^{26}$ showed that $C$. albicans was the most common vaginal Candida species, followed by $C$. glabrata as a second species causing vaginal candidiasis among pregnant women, but other species $\mathrm{s}$ uch as C. krusei, C. tropicalis and $C$. parapsilosis are also encountered. Yadav and Prakash ${ }^{27}$ reported high occurrence of C. albicans (64.04\%) followed by glabrata (12.35\%), dubliniensis (10.11\%), tropicalis (5.61\%) and C. krusei (3.37\%). Another study by Sutaria et al. ${ }^{28}$ reported C. albicans (35.55\%) as the most common vaginal Candida species, followed by C. glabrata $(9.63 \%)$, causing vaginal candidiasis among pregnant women. The high occurrence rate of $C$. albicansas a causative agent of vaginal candidiasis during childbearing age maybe due to its virulent factors, which include dimorphism and phenotypic switching. Also, C. albicans produces protease and phosphatase enzymes, where they enhance its attachment to the human epithelium. It can also be found that the high occurrence rate of $C$. albicans could be due to increased physiological changes, estrogen, and rich glycogen content of the vaginal mucosa, thereby providing an adequate supply of utilizable sugar that favor its growth during pregnancy. ${ }^{14}$ However, Ghaddar et al. ${ }^{7}$ reported a low incidence of $C$. albicans (42\%) comparing to non-albicans Candida species (58\%), where include C. glabrata and C. krusei. Molecular subtyping of C. albicans provides a useful tool for studying the epidemiology of this commonly causative agent of candidiasis in humans for identification of pathogenic subpopulation within this species, thereby assist in developing appropriate therapeutic strategies to combat the disease. ${ }^{13,29}$ In Iraq, there is insufficient data on vaginal candidiasis in pregnant women and the distribution of the vaginal Candida species; thus, this study considers as the first report of genotypic analysis of C. albicans isolated from vaginal candidiasis in pregnant women. In this study, 64 isolates of $C$. albicans were obtained from vaginal swabs in pregnant women, depending on the transposable intron in 25S rDNA all isolates were differentiated into three genotypes (A, B, and $C$ ), none of the isolates belonged to genotype $(E)$. The data revealed that the most prevalent genotype among all isolates was Genotype A (54.69\%), followed by Genotype B (34.38\%) and Genotype C (10.94\%). These results agree with those reported by previous studies, ${ }^{29,30}$ whereas, disagree with the results of Ali Shtayeh et al., ${ }^{31}$ who reported that the dominant genotype is Genotype C (55\%), followed by Genotype A (32.4\%), and Genotype B (12.6\%). The variation between three genotypes of $C$. albicans, may be due to the level of secretion and activity of exoenzymes such as phospholipase and proteinase since they are noteworthy virulence factors where contribute to the pathogenesis. ${ }^{32,33}$ 
Notably, there is limited information regarding phylogeny and genetic diversity of C. albicans strains isolated in vaginal candidiasis of pregnant women in Iraq. It is revealed that $C$. albicans population isolated from vaginal candidiasis in pregnant women despitethe genetic variation depending on $25 \mathrm{~S}$ rDNA, they are belonging to one clade (Figure 6). This clade of $C$. albicans was more similar to the $C$. albicans strain from Belgium (X53497.1), while genetically very different with strains from the Netherlands (MH545917.1), India (MT036888.1), Egypt (MK886759.1), and China(MN826341.1). The phylogeny results of three $C$. albicans genotypes are shown in Figure 7 , and it is found that genotypes $A$ and $B$ are more similar to each other, comparing to the genotype $\mathrm{C}$.

\section{Conclusion}

The study concluded a high prevalence of vaginal candidiasis, and vaginal infection among pregnant women by $C$. albicans dominates other non-albicans species such C. glabrata, C. kefyr, C. tropicalis, and C. krusei. Molecular genotyping revealed that three genotypes (A, B, and C) of $C$. albicans causing vaginal candidiasis in pregnant women. In addition, this study also found a genetic variation between C. albicans genotypes. Therefore, future works should focus on the correlation of genotypes to the host predisposing factors, pathogen virulence factors, and their antifungal susceptibility.

\section{Competing interests}

The author declares no competing interests.

\section{References}

1. Seyoum E, Bitew A, Mihret A. Distribution of Candida albicans and non-albicans Candida species isolated in different clinical samples and their in vitro antifungal suscetibity profile in Ethiopia. BMC Infect Dis 2020; 20(1):231.

2. Hedayati MT, Taheri Z, Galinimoghadam T, Aghili SR, Yazdani Cherati J, Mosayebi E. Isolation of different species of Candida in patients with vulvovaginal candidiasis from Sari,
Iran. Jundishapur J Microbiol 2015; 8(4).

3. Rathod SD, Klausner JD, Krupp K, Reingold AL, Madhivanan $P$. Epidemiologic features of vulvovaginal candidiasis among reproductive-age women in India. Infect Dis Obstet Gynecol 2012; 2012: 859071.

4. Lema VM. Recurrent vulvo-vaginal candidiasis: Diagnostic and management challenges in a developing country context. Obstet Gynecol Int J 2017; 7(5):00260 .

5. Oviasogie FE, Okungbowa FI. Candida species amongst pregnant women in benin city, nigeria: effect of predisposing factors. Afr J Cln Exper Microbiol 2009. 10(2):92-8.

6. Parveen N, Munir AA, Din I, Majeed R. Frequency of vaginal candidiasis in pregnant women attending routine antenatal Clinic. J Coll Physicians Surg Pak 2008; 18(3):154-7.

7. Ghaddar N, Anastasiadis E, Halimeh $R$, Ghaddar A, Dhar R, AlFouzan W, et al. Prevalence and antifungal susceptibility of Candida albicans causing vaginal discharge among pregnant women in Lebanon. BMC Infect Dis 2020; 20(1):32.

8. Green CB, Marretta SM, Cheng G, Faddoul FF, Ehrhart EJ, Hoyer LL. RT-PCR analysis of Candida albicans ALS gene expression in a hyposalivatory rat model of oral candidiasis and in HIV-positive human patients. Med Mycol 2006; 44(2):103-11.

9. Kim GY, Jeon JS, Kim JK. Isolation frequency characteristics of Candida species from clinical specimens. Mycobiology 2016; 44(2):99-104.

10. Vignesh KB, Amar KG, Swapna M, Easow JM. Isolation and identification of candida species from various clinical samples in a tertiary care hospital. International Journal of Research in Medical Sciences 2017; 5(8):3520.

11. Zeng X, Zhang Y, Zhang T, Xue Y, Xu H, An R. Risk factors of vulvovaginal candidiasis among women of reproductive age in Xi'an: A Cross-Sectional Study. Biomed Res Int 2018; 2018:9703754.

12. Zanni $P$, Bonfim-Mendonca PS, Negri $M$, Nakamura SS, Donatti L, Svidzinski TIE, et al. Virulence factors and genetic variability of vaginal Candida albicans isolates from HIV-infected women in the post-highly active antiretroviral era. Rev Inst Med Trop Sao Paulo 2017; 59:e44.

13. Tantivitayakul P, Panpradit N, Maudcheingka T, Klaophimai A, Lapirattanakul J. Genotyping of Candida albicans and Candida dubliniensis by 25S rDNA analysis shows association with virulence attributes in oral candidiasis. Arch Oral Biol 2019; 97:18-24.

14. Oyewole O, Okoliegbe I, Alkhalil S, Isah P. Prevalence of vaginal candidiasis among pregnant women attending Federal University of Technology, Minna, Nigeria, Bosso Clinic. Research Journal of Pharmaceutical, Biological and Chemical Sciences 2013; 4(1):113-20. 
15. Odds FC, Bernaerts R. CHROMagar Candida, a new differential isolation medium for presumptive identification of clinically important Candida species. J Clin Microbiol 1994; 32(8): 1923-9.

16. Madhavan $\mathrm{P}$, Jamal $\mathrm{F}$, Chong $\mathrm{PP}, \mathrm{Ng} \mathrm{KP}$. Identification of local clinical Candida isolates using CHROMagar Candida as a primary identification method for various Candida species. Trop Biomed 2011; 28(2):269-74.

17. Daef E, Moharram A, Eldin SS, Elsherbiny N, Mohammed M. Evaluation of chromogenic media and seminested PCR in the identification of Candida species. Braz J Microbiol 2014; 45(1):255-62.

18. Lau A, Sorrell TC, Lee O, Stanley K, Halliday C. Colony multiplex-tandem PCR for rapid, accurate identification of fungal cultures. J Clin Microbiol 2008; 46(12):4058-60.

19. Alshahni MM, Makimura K, Yamada T, Satoh K, Ishihara Y, Takatori K, et al. Direct colony PCR of several medically important fungi using Ampdirect plus. Jpn J Infect Dis 2009; 62(2):164-7.

20. Fujita SI, Senda Y, Nakaguchi S, Hashimoto T. Multiplex PCR using internal transcribed spacer 1 and 2 regions for rapid detection and identification of yeast strains. J Clin Microbiol 2001; 39(10): 3617-22.

21. Chang HC, Leaw SN, Huang $A H, W u T L$, Chang TC. Rapid identification of yeasts in positive blood cultures by a multiplex PCR method. J Clin Microbiol 2001; 39(10):3466-71.

22. Jordan JA. PCR Identification of four medically important Candida species by using a single primer pair. J Clin Microbiol1994; 32(12):2962-7.

23. Kanagal DV, Vineeth VK, Kundapur R, Shetty $H$, Rajesh A. Prevalence of vaginal Candidiasis in pregnancy among coastal south Indian women. Journal of Women's Health, Issues \& Care 2014; 3(6).

24. Alsharifi EA. Epidemiology of vaginal candidiasis among pregnant women attending Tikrit teaching hospital-Iraq. J Fac Med Baghdad 2017; 59(4):321-4.

25. Goncalves B, Ferreira C, Alves CT, Henriques M, Azeredo J, Silva S. Vulvovaginal candidiasis: Epidemiology, microbiology and risk factors. Crit Rev Microbiol 2016; 42(6):905-27.

26. Nelson M, Wanjiru W, Margaret MW. Prevalence of vaginal candidiasis and determination of the occurrence of Candida species in pregnant women attending the antenatal clinic of Thika district hospital, Kenya. Open Journal of Medical Microbiology 2013; 03(04):264-72.

27. Yadav K, Prakash S. Prevalence of vulvovaginal candidiasis in pregnancy. Global Journal of Medicine and Medical Sciences 2016; 4(1):10816.

28. Sutaria P, Cholera M, Donga SB. A prevalence study of vaginal candidiasis among pregnant women. International Journal of Advances in
Medicine 2019; 6(3):922.

29. Zhu X, Dong N, Wang Q, Cheng B, Zhu L, Tao J, et al. Genotyping and antifungal susceptibility of Candida albicans clinical isolates from Yangzhou region in China. African Journal of Microbiology Research 2011; 5(25).

30. Liu XP, Fan SR, Bai FY, Li J, Liao QP. Antifungal susceptibility and genotypes of Candida albicans strains from patients with vulvovaginal candidiasis. Mycoses 2008; 52(1):24-8.

31. Ali Shtayeh MS, Jamous RM, Alothman NHA, Abu Baker MH, Abu Zaitoun SY, Mallah OY, et al. Genotyping and antifungal susceptibility of Candida albicans strains from patients with vulvovaginal and cutaneous candidiasis in Palestine. African Journal of Microbiology Research 2015; 9(13):952-9.

32. Oliveira SKR, Anjos DCV, Gonçalves LHB, Ferro TAF, Monteiro SG, Silva Figueiredo PMS, et al. Prevalence and producation of enzymes by Candida isolates from vaginal secretion sampls. Revista de Patologia Tropical 2013; 42(2):16176.

33. Shirkhani S, Sepahvand A, Mirzaee M, Anbari K. Phospholipase and proteinase activities of Candida spp. isolates from vulvovaginitis in Iran. J Mycol Med 2016; 26(3):255-60. 